Published by Al-Nahrain College of Medicine P-ISSN 1681-6579

E-ISSN 2224-4719

Email: iraqijms@colmed-alnahrain.edu.iq

http://www.colmed-alnahrain.edu.iq

http://www.iraqijms.net

Iraqi JMS 2018; Vol. 16(1)

\title{
Genotyping of Listeria Monocytogenes in Iraqi Women with Spontaneous Abortion Using Pulse Field Gel Electrophoresis
}

\author{
Khalid W. Qassim ${ }^{1}$ MSc, Azhar A.F. AL Attraqchi² PhD, Yaarub I. Khatab FIBMS (Path) \\ ${ }^{1}$ Department of Pathology and Forensic Medicine, ${ }^{2}$ Dept. of Medical Microbiology, College of Medicine, Al-Nahrain \\ University, Baghdad, Iraq
}

\begin{abstract}
Background Listeria monocytogenes (L. monocytogenes) is a Gram-positive, facultative intracellular bacterial pathogen that can cause a severe invasive disease (listeriosis) mainly in pregnant women. Based on genetic content, $L$. monocytogenes can be divided into 3 lineages I, II and III. Several molecular methods have been developed to assist in the characterization of $L$. monocytogenes, macrorestriction analysis by pulse-field gel electrophoresis (PFGE) is one of the most used methods for the genotyping of $L$. monocytogenes.

Objective To determine the predominant genotype of $L$. monocytogenes isolated from clinical cases in a group of aborted Iraqi women.

Methods A study was designed and included 15 clinical isolates of $L$. monocytogenes and one isolate from locally made cheese. The PFGE protocol was performed as described by Graves and Swaminathan. Briefly, bacterial suspensions adjusted to optical density of 1.3 at $610 \mathrm{~nm}$ were embedded in $1.2 \%$ Sea Kem Gold agarose plugs. The lysed, washed five times and a $2 \mathrm{~mm}$ thick piece was cut, equilibrated and digested with $200 \mu \mathrm{l}$ of Ascl enzyme master mix at $37^{\circ} \mathrm{C}$ for $2 \mathrm{~h}$. The macrorestriction fragments were separated by electrophoresis on a CHEF-DRIIBIO-RAD. Images were analyzed with the software Bio Numerics Gel Compar II version 6.6.11 (Applied Maths).

Results All L. monocytogenes isolates displayed Ascl digestion profiles after incubation with the enzyme, about 6-12 majorfragments of 30 to $675 \mathrm{~kb}$ were obtained, sixteen isolates have been used in this study, however, 11 different Ascl profiles were encountered, the distribution of the isolates according to the restriction profiles showed that two profiles were the most predominant A, A2 with a percentage of similarity of $100 \%$.
\end{abstract}

Conclusion L. monocytogenes genotyping showed profiles that can be well used in tracing down infection source and outbreak of this bacterium. PFGE represents a great discrimination and reproducibility method for molecular sub-typing of $L$. monocytogenes and it is considered the gold standard.

Keywords Listeria monocytogenes, genotyping, placenta, aborted women, pulse field gel electrophoresis

Citation

Qassim KW, AL Attraqchi AAF, Khatab YI. Genotyping of Listeria Monocytogenes in Iraqi women with spontaneous abortion using pulse field gel electrophoresis. Iraqi JMS. 2018; Vol. 16(1): 8-13. doi: 10.22578/IJMS.16.1.3

List of abbreviations: $\mathrm{CHEF}=$ Contour-clamp homogeneous electric field, PFGE = Pulse field gel electrophoresis, RFLP = Restriction Fragment Length Polymorphism, UPGMA = Unweighted-pair group method with arithmetic averages

\section{Introduction}

$L$ isteria monocytogenes monocytogenes) is a Gram-positive intercellular, rod shaped, nonspore- forming, motile, facultative anaerobic, bacterium. It causes a severe invasive disease (1).

Listeriosis during pregnancy may lead to intrauterine infection, which may result in severe complications like preterm labor, spontaneous abortion, and stillbirth and or infection of the neonate which may result in high morbidity and mortality rate ${ }^{(2)}$. Of several molecular methods currently available like 
polymerase chain reaction (PCR), amplified fragment length polymorphism detection, DNA sequencing and others, macrorestriction analysis by pulse-field gel electrophoresis (PFGE) is one of the most used methods for the subtyping of $L$. monocytogenes. The using of restriction endonuclease $\mathrm{Ascl}$, as advised by Pulse Net USA, has shown excellent discrimination for $L$. monocytogenes and the technique is shown to be reproducible. PFGE is considered to be the international standard for subtyping ${ }^{(3)}$.

PFGE is a form of RFLP (Restriction Fragment Length Polymorphism) typing in which, bacterial genome is digested with a rare cutting restriction enzymes that cut the bacterial genomic DNA infrequently and therefore generate a small number of DNA fragments between (10-20 bands); these fragments are of a large sizes, from $20 \mathrm{~kb}$ to $10,000 \mathrm{~kb}$, and are separated using specific electrophoresis techniques (4) for $L$. monocytogenes, the enzymes are Ascl or Apal, these enzymes may generate between 6 to 12 and 14 to 17 fragments respectively separated through the PFGE. Combinations of the two profiles generated by the enzymes are used to characterize L. monocytogenes strains ${ }^{(5)}$.

Genetic comparisons among isolates carry out by using the differences in the restriction profiles. Computer-based analysis is very simple and enables rapid comparison on strains. Currently, PFGE represent the "gold standard" of the molecular typing techniques for foodborne pathogenic bacteria such as Salmonella, E. coli, Yersinia, Vibrio and Listeria (6).

The aim of this study and the benefit of it is to assess the extent of $L$. monocytogenes in causation of human spontaneous abortion in a sample of Iraqi women, and to determine the genetic subtypes of listeria monocytogenes associated with abortion.

\section{Methods}

A cross-sectional study was designed that included 250 placental tissues obtained from aborted women (which their acceptance was taken before sample collection) attended AlImamein AL-Kadhimein Teaching Hospital in Baghdad during the period from June 2014 to November 2015. Out of total number of the placentas samples, only fifteen isolates of $L$. monocytogenes were identified. The isolation and identification of $L$. monocytogenes were performed according to Collee et al. 1996 and McFaddin, $2000^{(7,8)}$.

\section{PFGE of L. monocytogenes}

The laboratory Protocol for molecular subtyping of $L$. monocytogenes by PFGE includes the following:

The Culture growth: An isolated colony from a pure culture of $L$. monocytogenes was streaked onto nutrient agar (NA) plates and the plates were incubated at $37^{\circ} \mathrm{C}$ for $14-18 \mathrm{~h}$.

Pulse field gel electrophoresis: The PFGE protocol was performed as described by Graves and Swaminathan (2001) ${ }^{(6)}$. Briefly, bacterial suspensions adjusted to optical density of 1.3 at $610 \mathrm{~nm}$ were embedded in $1.2 \%$ SeaKem Gold agarose plugs. The lysed, washed five times and a $2 \mathrm{~mm}$ thick piece was cut, equilibrated and digested with $200 \mu \mathrm{l}$ of Ascl enzyme master mix at $37{ }^{\circ} \mathrm{C}$ for $2 \mathrm{~h}$. The macrorestriction fragments were separated by electrophoresis on a CHEF-DR II BIO-RAD (USA) in a $1 \%$ Pulse Field Certified Agarose gels (Ultrapure DNA grade agarose) at Initial switch time: $4.0 \mathrm{~s}$; Final switch time: $40.0 \mathrm{~s}$; Voltage: 6 V; Included Angle: $120^{\circ}$ Run time: $18-19$ hours.

Staining and Documentation of an Agarose gel: After electrophoresis run was over, gel was removed and stained with ethidium bromide for 20-30 min in covered container. The use of restriction endonucleases $\mathrm{Ascl}$, is advised by Pulse Net USA, and has shown excellent discrimination for L. monocytogenes. Images were analyzed using Gel Compare II evaluation software version 6.6.11 from Applied Maths.

The similarity between fingerprints was determined using number of different bands coefficient with a $1 \%$ tolerance between band positions. The cluster analysis and generation of Dendrogram was performed using 
Unweighted-pair group method with arithmetic averages (UPGMA) with a similarity score value of $100 \%$ as the cutoff.

\section{Results}

All isolates of Listeria monocytogenes were digested with Ascl restriction endonuclease...GG $\nabla$ CGCGCC... .

...CCGCGC $\Delta \mathrm{GG} . .$.

Only one was isolated from local made cheese, which presented in lane number 1 (Figure 1). Isolates of L. monocytogenes were typed using PFGE. Analysis of the Ascl restriction profiles by automated cluster analysis was done using number of different bands as a similarity coefficient and tolerance: $1 \%$.
All the isolates displayed Ascl digestion profiles after incubation with the enzyme (Figure 1), about 6-12 major fragments of 30 to $675 \mathrm{~kb}$ were obtained following the digestion using Ascl.

Sixteen isolates have been used in this study, fifteen represent the clinical isolates and the

Eleven PFGE types and two subtypes were distinguished with the restriction endonuclease Ascl through visual inspection, using Unweighted-pair group method with arithmetic averages (UPGMA) with a similarity score value of $100 \%$ as the cutoff.

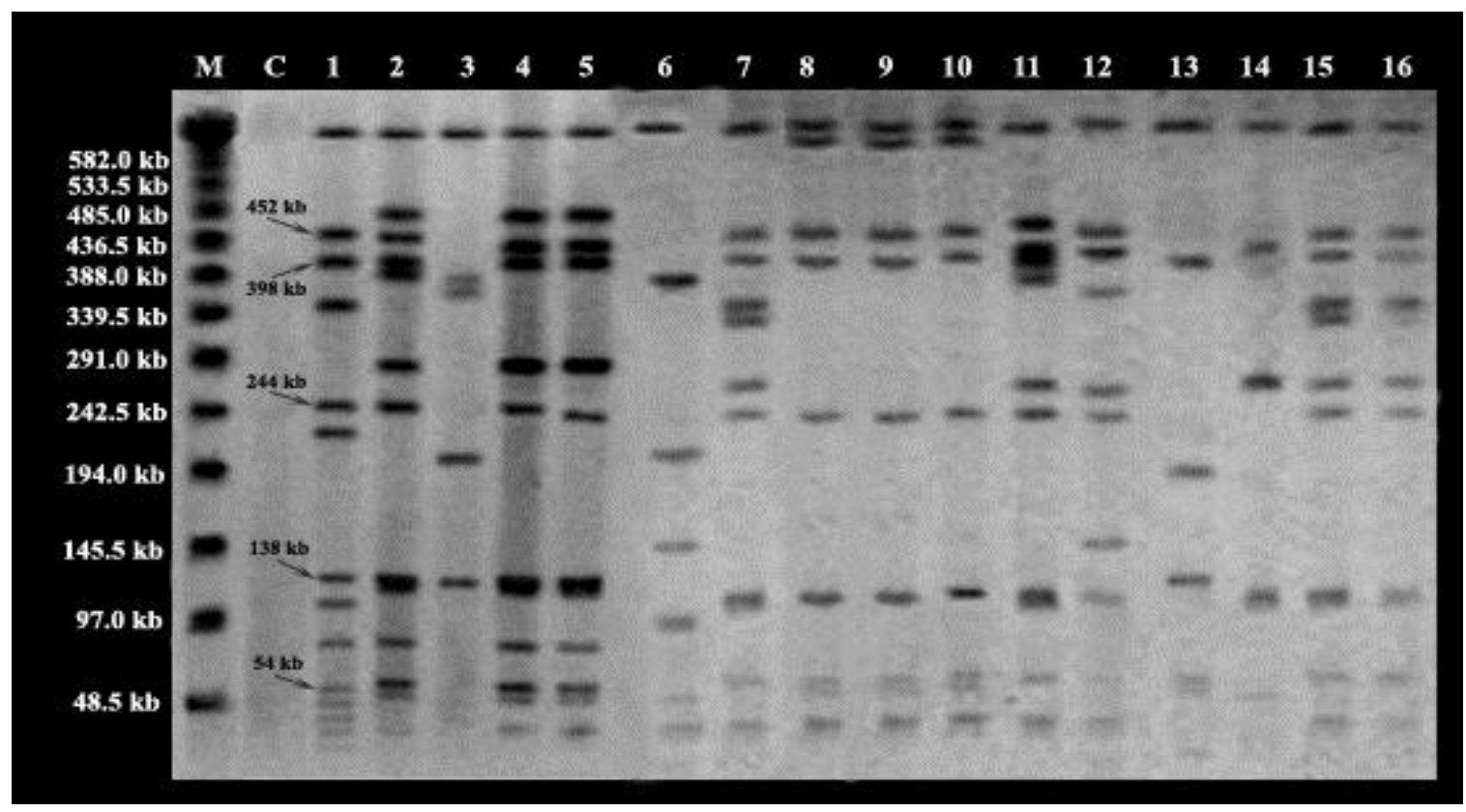

Figure 1. Genomic DNA restriction patterns of Listeria monocytogenes isolates, performed by PFGE after digestion with Ascl

Cluster analysis of the Ascl restriction digest profiles showed that PFGE subtype $A$ and subtype A2 were predominant with a percentage of similarity of $100 \%$ for both followed by type $A 3$ with a percentage of similarity of $99 \%$ then $A 5$ and $B$ with a percentage of similarity of $98 \%, D$ and $A$ of
$97.5 \%, A 4$ and $A 5$ of $97 \%, A 1$ of $94.5 \%, F$ and $E$ of $93.5 \%$, I was of $93 \%$, and $G$ was presented with a percentage of similarity of $90 \%$. Cluster analysis of L. monocytogenes isolates and the PFGE types assigned by visual inspection and by automated cluster analysis are shown in (Figure 2). 


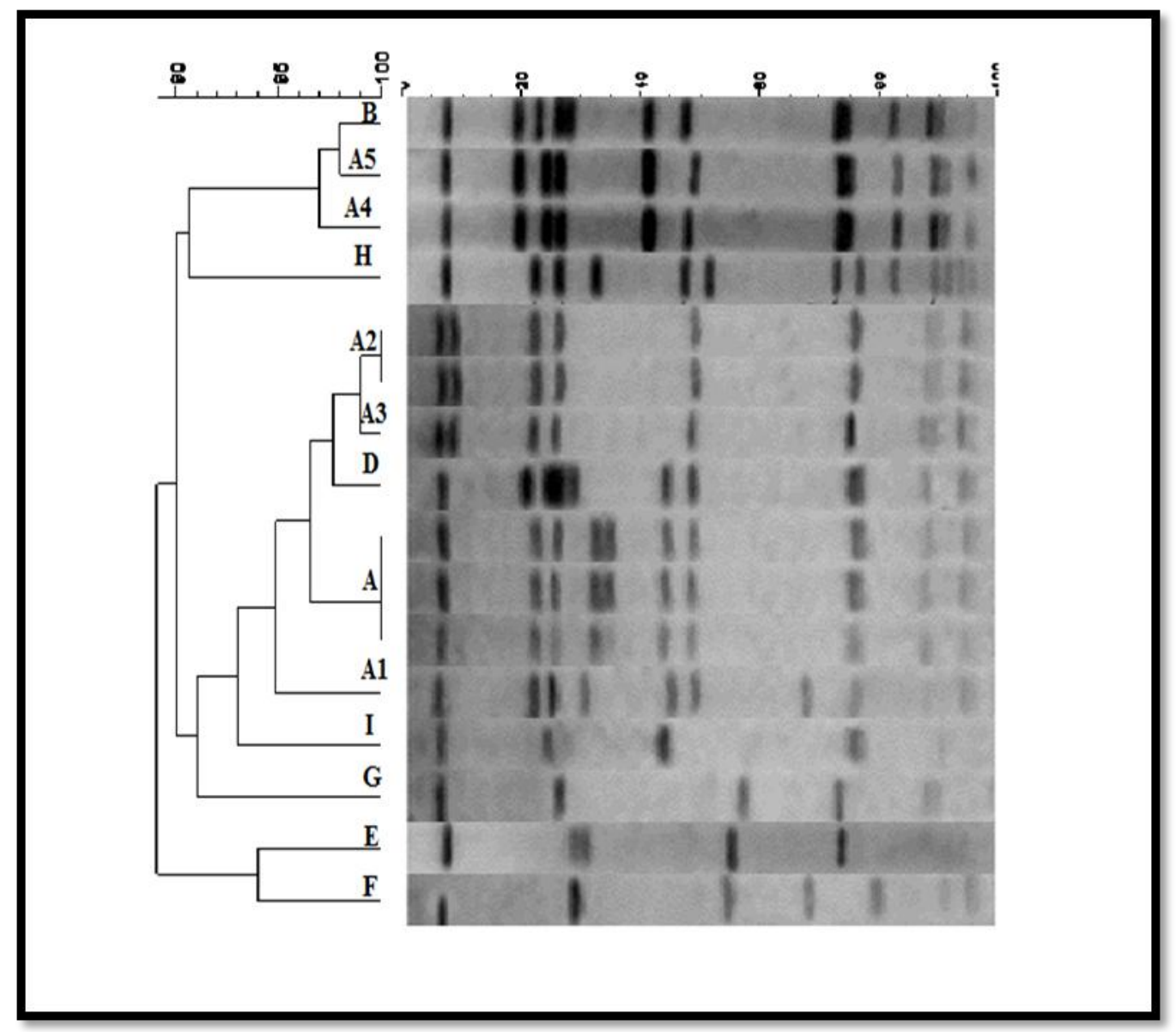

Figure 2. Dendrogram representing PFGE types assigned by Automated Cluster Analysis (ACA) of the Ascl restriction digest profiles of Listeria monocytogenes isolates

\section{Discussion}

The large number of different PFGE types (sixteen isolates yield eleven PFGE types) may indicate that a variety of genetically distinct strains cause listeriosis in Iraq, however, this high number of PFGE types is in line with several published data for clinical cases $(9,10)$, this variation in the number of PFGE types obtained in different studies may be due to that the clinical strains used in these studies not corresponded to sporadic cases; the choice of the rare cut endonucleases; and the analysis protocol used in the study. However, the current work was done using the Pulse Net method described by Graves and Swaminathan (6).

The cluster analysis results showed that there was two predominant subtypes $A$ and $A 2$, which include three isolates and two respectively, those isolates were genetically indistinguishable from each other and also genetically related to other subtypes and or PFGE types with a percentage of similarity range from $99 \%$ to $89 \%$, however those with high similarity may differ in a single band only while the lower similarity percentage was associated with isolates differ in more than one band.

Although PFGE profiles give a considerable degree of strain diversity in L. monocytogenes populations, the number of bands differences required for discrimination between strains is controversial ${ }^{(11)}$. Strains differing by more than one band are clearly distinct but some strains from geographically distinct sources may exhibit few or no differences in DNA profile and by the Turnover criteria could be considered epidemiologically related (12). According to 
Autio et al. (2002) (13), the isolation of indistinguishable PFGE types may mislead the investigation towards establishing the vehicle or the source of infection. However, many food products may carry genetically identical strains without any cross-contamination or common infection sources. Therefore, a suspected epidemic infection may consist of two or more independent sporadic cases.

In conclusion, human listeriosis in Iraq involves a great variety of genetically different isolates. However, in this study, indistinguishable isolates were isolated from more than one patient, suggesting the existence of more than one episodes of listeriosis. In all of the listeriosis episodes, the food vehicle was not determined, because of a lack of epidemiological evidence.

\section{Acknowledgments}

The authors are deeply thankful to the staff of Laboratory of Medical Microbiology Department and the staff of Laboratory of Pathology Department in the Medical College of Al-Nahrain University for their valuable support and advice. The authors also would like to thank Dr. Saad Sabah and Mr. Farked Farhan in Ministry of Science and Technology, for their continuous support in providing a suitable environment to finish my research.

\section{Authors Contribution:}

Qassim collected the cases, performed the bacterial tests and analyzed the results. Dr. AL Attraqchi helped in the study design and supervising the work. Dr. Khatab participated collection of cases and performed the histopathological examination.

\section{Conflict of interest}

The authors declare no conflict of interest.

\section{Funding}

This research was funded by College of Medicine/Al-Nahrain University.

\section{References}

1. Nes FD, Riboldi GP, Frazzon AP, et al. Antimicrobial resistance and investigation of the molecular epidemiology of Listeria monocytogenes in dairy products. Rev Soc Bras Med Trop. 2010; 43(4): 382-5. doi: 10.1590/S0037-86822010000400009.

2. Jahangirsisakht A, Kargar, M, Mirzaee, A, et al. Assessing Listeria monocytogenes hly $A$ gene in pregnant women with spontaneous abortion using PCR method in Yasuj, south west of Iran. Afr J Microbiol. Res. 2013; 7(33): 4257-60. doi: 10.5897/AJMR12.1484.

3. Roussel S, Félix B, Grant $K$, et al. Fluorescence amplified fragment length polymorphism compared to pulsed field gel electrophoresis for Listeria monocytogenes subtyping. BMC Microbiol. 2013; 13: 14. doi: 10.1186/1471-2180-13-14.

4. Herschleb J, Ananiev G, Schwartz DC. Pulsed-field gel electrophoresis. (2007). Nat Protoc. 2007; 2(3): 67784. doi:10.1038/nprot.2007.94.

5. Carriere C, Allardet-Servent A, Bourg G, et al. DNA polymorphism in strains of Listeria monocytogenes. J Clin Microbiol. 1991; 29(7): 1351-5.

6. Graves LM, Swaminathan B. PulseNet standardized protocol for subtyping Listeria monocytogenes by macrorestriction and pulsed-field gel electrophoresis. Int J Food Microbiol. 2001; 65(1-2): 55-62. https://doi.org/10.1016/S0168-1605(00)00501-8.

7. Collee JG, Fraser AG, Marmion BP, et al. Mackie and McCartney Medical microbiology. $14^{\text {th }}$ ed. USA: Churchill Living Stone. Inc; 1996.

8. McFaddin JF. Biochemical tests for identification of medical bacteria. $1^{\text {st }}$ ed. Baltimore USA: The Williams and Wilkins; 2000.

9. Gianfranceschi M, Pourshaban M, Gattuso A, et al. Characterization of Listeria monocytogenes strains isolated from food and humans in Italy by pulsedfield gel electrophoresis. Food Microbiol. 2002; 19(1): 47-55. doi: https://doi.org/10.1006/fmic.2001.0463.

10. Lozniewski A, Humbert A, Corsaro D, et al. Comparison of sludge and clinical isolates of Listeria monocytogenes. Lett Appl Microbiol. 2001; 32(5): 336-9. doi: 10.1046/j.1472-765X.2001.00918.x.

11. Brosch R, Brett $M$, Catimel $B$, et al. Genomic fingerprinting of 80 strains from the WHO multicentre international typing study of Listeria monocytogenes via pulsed-field electrophoresis (PFGE). Int J Food Microbiol. 1996; 32(3): 343-55. doi: https://doi.org/10.1016/S0168-1605(96)011476.

12. Tenover FC, Arbeit RD, Goering RV, et al. Interpreting chromosomal DNA restriction pattern produced by Pulsed-field gel electrophoresis: Criteria for bacterial strain typing. J Clin Microbiol. 1995; 33(9): 2233-9.

13. Autio $T$, Lundén J, Frederiksson-Ahomaa $M$, et al. Similar Listeria monocytogenes pulse types detected in several foods originating from different sources. Int J Food Microbiol. 2002; 77, 83-90. doi: http://dx.doi.org/10.1016/S0168-1605(02)00055-7. 
Iraqi JMS 2018; Vol. 16(1)

Correspondence to Dr. Azhar A.F. AL Attraqchi

Received Jan.11 ${ }^{\text {th }} 2017$

E-mail: tariq_963@yahoo.com

Accepted May.24 2017

dr.azhar.ibrahim@colmed-alnahrain.edu.iq 